\title{
Slicing strategies to obtain accuracy of feature relation in rapidly prototyped parts
}

\author{
Y.Y. Chiu ${ }^{a, *}$, Y.S. Liao ${ }^{\text {a,b }}$, S.C. Lee ${ }^{a, b}$ \\ a Department of Industrial and Commercial Design, Oriental Institute of Technology, 58, Sec. 2, Syh-Chuan Rd., Baan-Chyan, Taipei 220, \\ Taiwan, ROC \\ b Department of Mechanical Engineering, National Taiwan University, Taipei 106, Taiwan
}

Received 21 August 2003; received in revised form 16 December 2003; accepted 14 January 2004

\begin{abstract}
A new slicing method and its associated algorithm to ensure unilateral tolerance over the whole prototype are proposed in this paper. The proper slicing rule is selected according to the inner product of the part's normal vector on STL file and working direction, together with the desired function of the part to be manufactured. To reduce error, proper solutions for geometric shapes that lose their qualities easily, such as peaks, valleys, flat areas and fine features, are presented. In addition, the amounts of tolerance volume and effective cusp are computed to facilitate post-processing of the part. Finally, slicing procedures are proposed and experimentally verified.
\end{abstract}

(C) 2004 Elsevier Ltd. All rights reserved.

Keywords: Slicing; Rapid prototyping; Algorithm

\section{Introduction}

Based on the principle of layer manufacturing, the rapid prototyping $(\mathrm{RP})$ technique starts with the intersection of the 3D model from CAD (usually an STL file) with layers of $2 \mathrm{D}$ horizontal planes. As a result, a stack of 2D geometry contours is obtained, each representing a cross-section of the 3D model. Next, the raw material is placed on the work table. The computer takes the bottom slice of the 3D model and transmits different levels of energy to the raw material to the location as designated by the geometric contour. The raw material is filled in one slice after another from the bottom-up, and the process is repeated until a complete 3D part is produced. Theoretically, given an infinitely small thickness of each $2 \mathrm{D}$ slice, the $3 \mathrm{D}$ part should be identical with the 3D model constructed by the computer. However, in reality, materials used in RP are all limited in thickness, thus generating a staircase error. Besides, there are also other factors that

\footnotetext{
${ }^{*}$ Corresponding author. Fax: +886-2-29544672.

E-mail address: petecyy@ms19.hinet.net (Y.Y. Chiu).
}

may affect RP precision. These errors have been discussed in great detail in related studies [1,2], and they are not repeated here.

The part produced by RP presents a stepwise shape, which is more evident as the thickness of the $2 \mathrm{D}$ slices increases. Precision becomes inferior when the workpiece counter slope is gentle. On the contrary, machining precision becomes better as the workpiece contour slope becomes steeper. Variation of the shape of a part is thus an important factor in precision. In addition, the presence of fine features in the workpiece smaller that the thickness of a layer affects the machining precision as well. Current studies on the improvement of precision are discussed in the following sections.

Dolenc and Kulkarni [3,4] used the original CAD file in the study of slicing methods. The former searched for optimal slicing thickness for a variety of workpiece features. The latter derived the optimal slicing thickness based on the workpiece shape curvature and the cusp height between layers. Sabourin et al. [5,6] divided a workpiece into the "outer region" in contact with the boundary and the "inner region" without contact with the boundary. The "outer region" affects precision. 
Thus, a variety of slicing thicknesses is applied based on the variation of workpiece contour shape. A thinner thickness is used for a workpiece of smoother contour slope. In contrast, greater thickness is used when the workpiece counter slope becomes steeper. As for the "inner region", since it does not affect precision, its thickness is greater than that of the "outer region" to increase machining efficiency.

Hope et al. [7] presented a TruSurf system using a five axis numerical control (NC) waterjet cutter machine to cut the layers. Inclined surfaces are obtained by controlling the orientation of the waterjet cutter used to eliminate the staircase shape so as to reduce the error size. Later on, Hope et al. [8] used this system and took the mid-point of a layer in a workpiece of curved shape as the basis for calculating the machining contour and deciding the slope of the cutting vector. This proposed method increases accuracy but makes machining complicated.

The approaches adopted in previous studies all focus on the premise of minimizing the tolerance. They all overlook the importance of tolerance uniformity. Such negligence often results in the two annoying problems of over-cut and under-cut in the same workpiece. That is, the position of tolerance cannot be ascertained in a finished workpiece, and this leads to difficulty in postprocessing. From the perspective of post-processing, which is essential., a proper working tolerance is necessary for RP. If all tolerance is left outside the original workpiece contour, it is called "positive tolerance". On the contrary, when all tolerance is left inside the original workpiece contour, it is called "negative tolerance". In other words, positive tolerance leaves extra material, and thus is also called "over-size", as shown in Fig. 1(a). Negative tolerance leads to material insufficiency, and is thus called "under-size", as shown in Fig. 1(b). From a practical viewpoint, both types of tolerance have their specific usages. For most RP applications, such as industrial mock-up and investment casting, positive tolerance is necessary because the material left can be eliminated in post-processing to meet precision requirements. However, for certain industrial applications such as the cores used in producing cavities, no positive tolerance is required. Instead, over-cut is needed to make the finished product smaller than the actual dimensions so that the (a)

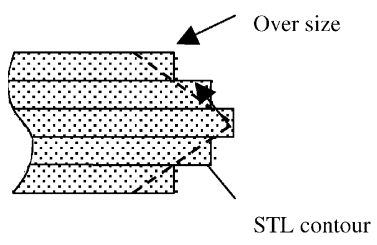

(b)

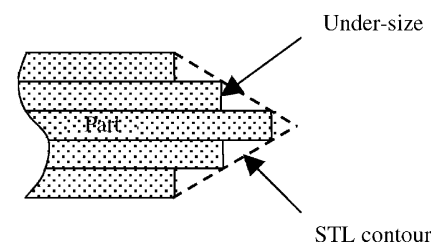

Fig. 1. Illustration of cases of (a) over-size and (b) under-size. duplicated cavity has extra tolerance for post-processing. Hence, a negative tolerance is needed in this case. These examples all represent important factors to be considered for RP improvement, which, unfortunately, have always been ignored.

From another perspective, in most RP techniques, limited by the consideration of working efficiency or process technologies, uniform slicing is the most commonly adopted basic setting to meet the requirement of working efficiency. A material thickness around 0.1 $\mathrm{mm}$ is commonly used at present. Take the RP of gluing sheets (e.g. laminated object manufacturing process) as an example. Since sheets of uniform thickness are used as the material for machining, material thickness cannot be changed according to variations in the features of a part's shape. Hence, this approach easily loses fine features and further affects the dimensional accuracy. Other powdered or liquid RP also use materials of uniform thickness in most cases to increase working efficiency, and hence the same problems are produced.

In addition, some special geometric features will be lost during the current slicing method. If parts have such features, such as peaks or valleys that are to be horizontal, these features may be easily lost during the slicing process. The example is shown in Fig. 1(b): the dashed line is the original STL contour and the staircase line is the actual slicing result; the peak feature is lost apparently. If a part has a flat area or something close to a flat area, there is also the problem of losing features during slicing. In the case of parts with fine features, especially when the size of these features is smaller than the distance between slices, these features are also easily lost.

Regardless of the slicing method of positive tolerance or negative tolerance, the finished part requires further post-processing such as sanding to obtain a refined surface and accurate precision. Hence, information on the tolerance volume and effective cusp needed for post-processing will be extremely helpful for those involved in post-processing.

Based on the above review, it is seen to be important to achieve unilateral tolerance over the whole prototype under the guideline of ensuring precision and retaining fine features; slicing methods which preserve special geometric features are developed in the paper. Unilateral tolerance here means keep tolerance on the same side for the whole prototype, either outside the part or inside the part. In this paper, a new slicing rule for uniform thickness to ensure that the entire workpiece possesses either positive or negative tolerance is developed, especially for such geometric shapes as peaks, valleys, flat areas and fine features, which easily lose their identity, and the amounts of tolerance volume and effective cusp are shown for the post-process procedure. The method proposed in this paper can be 
applied in a variety of RP systems, especially for the cases of uniform slice thickness commonly used.

\section{Slicing strategies}

The current RP technique adopts two strategies when using the slicing method to produce a 3D part. The most common approach is top-down slicing. In this method, the intersection point between the triangle facet and slicing plane is calculated and extended down to the thickness of the layered material, as shown in Fig. 2(a). These steps are repeated to form a 3D part. The other is bottom-up slicing, which extends from the intersection point up to the thickness of the layered material. From another perspective, it can be explained as the extension up from the intersection point between the slicing plane of the previous layer and triangle facet to the thickness of the layered material, as shown in Fig. 2(b). Repeating these steps can also form a 3D part. Calculations of the intersection point for both methods can be found in the literature [9].

\subsection{Calculation methods of top-down and bottom-up slicing}

Fig. 2 shows an elevational cross-sectional view of the interface between the part and the waste material. In the figure, there are two different regions. The area enclosed by the dashed line is the cross-section of a $3 \mathrm{D}$ part. The dotted area is the cross-section of the part after the slicing of layers. As shown in Fig. 2(a) and (b), due to slope variations, both the top-down slicing method and bottom-up slicing method result in conditions that are out of control, namely "under-size" and "over-size", respectively. It will be impossible to

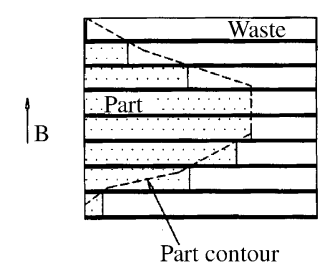

(a) Top-downslicing

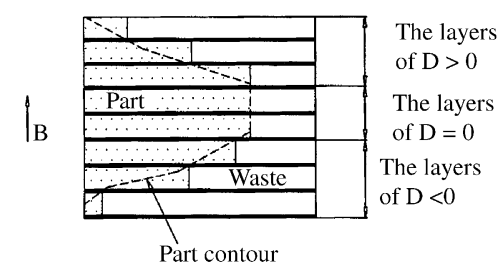

(c) Positive tolerance slicing

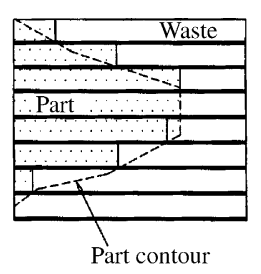

(b) Bottom-up slicing

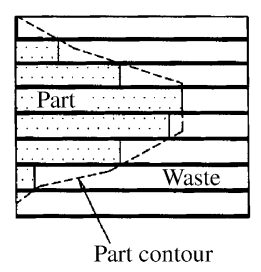

(d) Negative tolerance slicing
Fig. 2. An elevational cross-sectional view of the interface between the part and the waste material. This is a case of RP of gluing sheets. obtain accurate shape and satisfactory results by sanding the resulting part. In other words, the popular topdown slicing method can create "under-size" or "oversize" problems depending on the slopes given. With a negative slope, vertical cutting by laser along the intersection point results in under-size. On the other hand, with a positive slope, vertical cutting by laser along the intersection point results in over-size. The exact size can be obtained only for a region with vertical sides. In comparison, under-size makes the machined size smaller than the designed value, while over-size implies extra material after rapid prototyping. As discussed earlier, both methods have their own unique applications. From the above discussions, an attempt to place all tolerance on the same side is essential from a practical needs viewpoint. The engineer can choose the type of tolerance depending on the workpiece application, as recommended before applying rapid prototyping.

\subsection{Slicing strategies for positive tolerance and negative tolerance}

Generally speaking, geometric information of a 3D part cannot be fully described by the slope of the part alone. Decisions made based only on the part's slope may possibly lead to opposite results. For example, when the part and the waste material are exchanged, as shown in Fig. 2(a) and (b), the originally expected over-size turns into under-size, leading to the opposite results of the original expectation, and vice versa. This is an obvious example. Feygin and Pak [10] developed a slicing rule based on the positive or negative value of the part's slope to ensure the presence of extra working tolerance for post-processing. However, this can easily result in the above-mentioned ambiguous conditions. Thus, the method based on the part's slope is not appropriate. To solve this problem, positive tolerance slicing method and negative tolerance slicing method are proposed, and the results of slicing are shown in Fig. 2(c) and (d), respectively.

As mentioned earlier, an STL file is capable of describing the normal vectors and the coordinates of three vertices of a triangular facet, as shown in Fig. 3(a). Referring to Fig. 3, let the unit vector of RP working direction be $\boldsymbol{B}$, the normal vector of each triangle face be $\xi$, and the angle between each triangle face and horizontal axis be $\theta$. The user determines the preferred orientation based on such factors as machining precision, machining time, part strength and difficulty in waste material removal (for LOM). Next, the orientation of each triangular face is calculated based on the inner (dot) product value of the normal direction of each triangle face and RP working direction (B). That is, Eq. (1) denotes the results derived from 


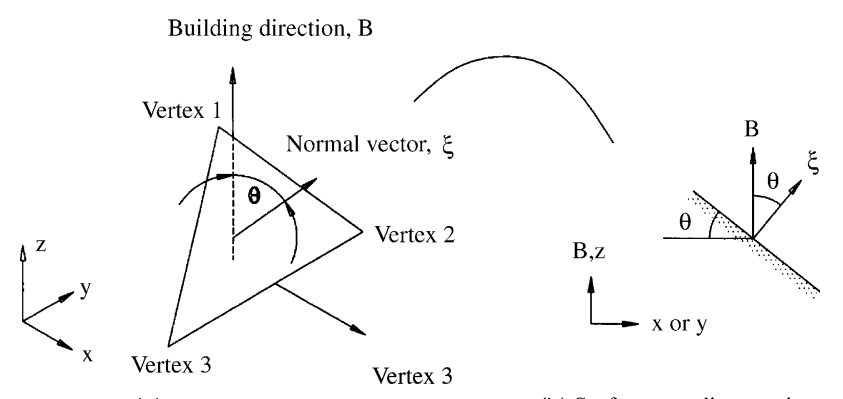

$\begin{array}{ll}\text { (a) Triangular facet } & \text { (b) Surface coordinate axis }\end{array}$

Fig. 3. Schematic diagram of triangular facet and working direction. $\xi$ is the normal vector of the facet; $B$ is the working direction.

the generally used top-down slicing method.

$\vec{\xi} \cdot \vec{B}=D$,

where $\xi=n_{i} \vec{i}+n_{j} \vec{j}+n_{k} \vec{k}$ is the normal vector of each triangle face in the STL file, and $\vec{B}$ is the working direction of RP process. Then,

if $D=0$, the intersection point on the $z$ axis is the exact contour;

if $D<0$, the intersection point on the $z$ axis is the positive tolerance;

if $D>0$, the intersection point on the $z$ axis is the negative tolerance.

Hence, if $\boldsymbol{D}=0$ during the slicing process, it means that the contour is vertical. In other words, the contour of the intersection point overlaps with the original contour. It maintains the exact contour without producing any error. If $\boldsymbol{D}<0$ (a positive slope in the case of Fig. 3), it implies that the angle between the contour and working direction is smaller than $90^{\circ}$, and cutting along the intersection point contour naturally forms a positive tolerance. If $\boldsymbol{D}>0$ (a negative slope in the case of Fig. 3), it implies that the angle between the contour and working direction is larger than $90^{\circ}$, and cutting along the intersection point contour causes a negative tolerance.

Therefore, if the user requires a positive tolerance and ends up with $\boldsymbol{D}>0$ in the calculation, the original intersection point should be replaced by the intersection point of the previous layer $(z=$ slicing height - one layer thickness), i.e. the bottom-up slicing method mentioned earlier. On the contrary, top-down slicing should be used to ensure extra working tolerance and avoid the problem of over-cut if $\boldsymbol{D}<0$. When $\boldsymbol{D}=0$, it implies a correct contour; then, both the top-down and bottom-up slicing method result in the same intersection point. The method is expressed in Eq. (2). Similarly, when a negative tolerance is required and if $\boldsymbol{D}<0$, then the bottom-up slicing method should be used. The use of the top-down slicing method ensures over-cut if $\boldsymbol{D}>0$. Again, $\boldsymbol{D}=0$ implies a correct contour, and either the top-down or bottom-up slicing method can be employed. The negative tolerance method is summarized and given in Eq. (3).

\section{Positive tolerance}

if $(D \geq 0) \quad$ calculate intersection point by

"Bottom-up slicing method" else calculate intersection point by "Top-down slicing method"(2)

Negative tolerance

if $(D \geq 0) \quad$ calculate intersection point by

"Top-down slicing method" else calculate intersection point by "Bottom-up slicing method"

\section{Slicing strategies for the special features}

\subsection{Peaks and valleys}

If parts have such features as peaks or valleys and these features tend to be vertical, no difficulty would encounter since the situation is similar to that of the inclined part discussed in the previous section. However, if the peaks or valleys are close to being horizontal, these features may be easily lost during the slicing process, as shown in Fig. 4.

How does one determine peaks or valleys? The answer can be discerned from the change of sign of the $\boldsymbol{D}$ value. When slicing occurs from the smallest $Z$ value upward, peaks occur at the layer where the $\boldsymbol{D}$ value changes from a negative value to a positive one, while valleys occur at the layer where a positive $\boldsymbol{D}$ value turns into a negative one. Regardless of the situation, the layer where change occurs (i.e. the layer where the $\boldsymbol{D}$ value changes sign) must be identified. The slicing thickness is set smaller ("fine slice") than usual. This thickness is $\Delta l$, as shown in Fig. 4(a). Then the proper intersection point is determined based on the realistic need of positive tolerance or negative tolerance. A feasible method is to take any one point $\mathrm{P}$ at the locations of fine slice $(i)$ and fine slice $(i+1)$ of the part. As for peaks, if positive tolerance is needed, calculate the horizontal distance between $\mathrm{P}$ and the intersections of

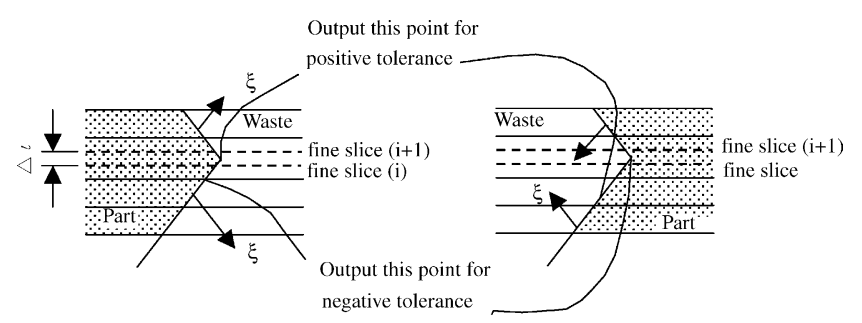

(a)

(b)

Fig. 4. Calculation of intersection point of (a) peak and (b) valley. 
fine slice $(i)$ and the horizontal distance between $\mathrm{P}$ and the intersections of fine slice $(i+1)$, then take the maximum value, which is the value sought for. In other words, the purpose of deciding the maximum value of fine slice $(i)$ and fine slice $(i+1)$ is to derive the intersection point value of the positive tolerance. On the contrary, if negative tolerance is needed, calculate the horizontal distance between $\mathrm{P}$ and the intersections of fine slice $(i)$ and the horizontal distance between $\mathrm{P}$ and the intersection of fine slice $(i+1)$, then take the minimum value.

As for the case of valleys, similar procedures may apply.

\subsection{Flat areas}

If a part has a flat area or some portion of the part close to a flat area, there is the problem of losing features during the slicing process, as shown in Fig. 5. When the ordinary top-down slicing method is used and the normal vector of the flat area is close to the working direction of RP, B, negative tolerance easily occurs. If the normal vector of the flat area forms an angle of approximately $180^{\circ}$ with the working direction of RP, positive tolerance takes place easily. Similarly, if the bottom-up method is used as the slicing rule, and the normal vector of the flat area is close to the working direction of RP, positive tolerance easily occurs. If the normal vector of the flat area forms an angle of approximately $180^{\circ}$ with the working direction of RP, negative tolerance easily occurs. The cause of this kind of problem is that certain triangle facets of the part are too flat (i.e. level plane or close to level), which is easily overlooked during the slicing process. Eq. (1) is also used to judge this situation. The inner (dot) product value of the normal direction of each triangle face and the RP working direction $(\boldsymbol{B})$ is employed to solve the problems. A feasible approach is described below.

When the above conditions are met during the slicing process, it means that feature loss exists. In this case, use the $\boldsymbol{D}$ value obtained from the inner product of the unit normal direction $(\xi)$ of the triangle facet and unit RP working direction $(\boldsymbol{B})$ for judgment. When $\boldsymbol{D}=-1$ and top-down slicing is used, an extra area will be added to the flat area, forming a positive tolerance. If bottom-up slicing is used, an area will be reduced from the flat area, forming a negative tolerance. In the same vein, if $\boldsymbol{D}=1$ and top-down slicing is used, an area will be reduced from flat area, forming a negative tolerance. In contrast, if bottom-up slicing is used, an extra area will be added to the flat area, forming a positive tolerance. Fig. 5(b) shows the case of positive tolerance slicing, while Fig. 5(c) shows that of negative tolerance slicing.

Therefore, based on user needs, if positive tolerance is required, top-down slicing should be used if $\boldsymbol{D}=-1$, and bottom-up slicing if $\boldsymbol{D}=1$. On the contrary, when negative tolerance is called for, bottom-up slicing should be used when $\boldsymbol{D}=-1$, while top-down slicing should be used when $\boldsymbol{D}=1$.

\subsection{Fine features}

In case of parts with fine features, especially when the size of these features is smaller than the thickness of each slice, these features are easily lost. The procedure to be followed is as follow:

1. The slicing thickness is set to a smaller value, called "fine slice", to calculate the intersection point. When slicing occurs from the smaller $Z$ value upward, the fine feature occurs at the layer where the $\boldsymbol{D}$ value changes sign.

2. After the fine feature occurs, by using the intersection point of the "fine slice" instead of that of the uniform thickness slice, one can reduce errors to a minimum.

\section{Calculation of tolerance volume and effective cusp height for post-processing}

\subsection{Calculation of tolerance volume}

Regardless of the slicing method of positive tolerance or negative tolerance, the finished part requires further post-processing, such as the sanding process, to obtain a refined surface and precision. Hence, information on the tolerance volume needed for post-processing will be extremely helpful for those involved in post-processing. The calculations of various types of tolerance volume are described in the following sections as shown in Fig. 6. (a)

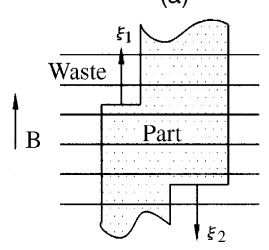

(b)

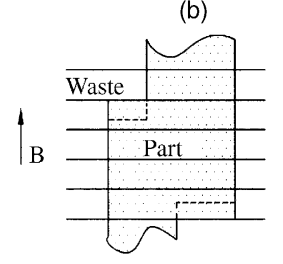

(c)

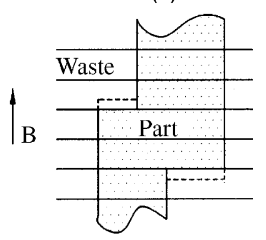

Fig. 5. (a) The flat area of the part before slicing; (b) positive tolerance slicing; (c) negative tolerance slicing. 


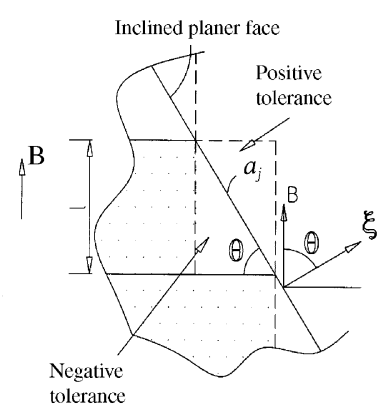

(a) Inclined planar face

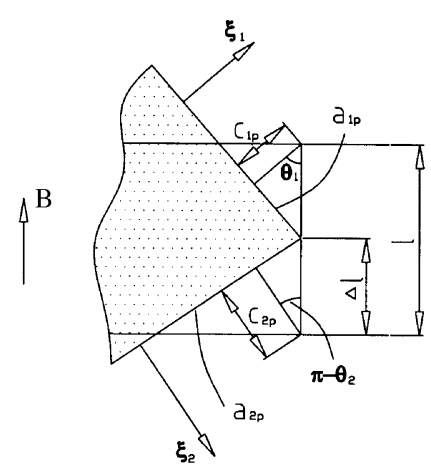

(b) Peak
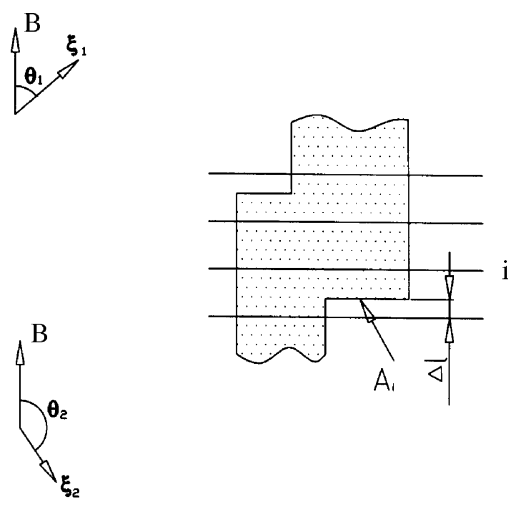

(c) Flat area

Fig. 6. Tolerance volume of the different special geometric features.

\subsubsection{Tolerance volume of the inclined planar face}

The equations below are employed to calculate the tolerance volume of an inclined planar face, where Eq. (4.1) is based on the unit of a layer and Eq. (4.2) is based on the unit of a triangle facet, as shown in Fig. 6(a):

$$
\begin{aligned}
\text { Tolerance volume } & =\sum_{i=1}^{L}\left(\sum_{j=1}^{M} 0.5 \cdot l \cdot a_{j} \cdot\left|\cos \theta_{j}\right|\right) \\
& =\sum_{k=1}^{N} 0.5 \cdot l \cdot A_{k}\left|\cos \theta_{k}\right|
\end{aligned}
$$

where $\cos \theta=(\vec{B} \cdot \vec{\xi}) /|\vec{B}||\vec{\xi}|$ where $l$ is the layer thickness, $\theta$ is the angle of inclination of the triangle facet, $i$ is the $i$ th layer, and $L$ is the total number of layers, $a_{j}$ is the area of the $j$ th inclined triangle face constrained in the $i$ th layer thickness, $A_{k}$ is the area of the $k$ th inclined triangle face, $j$ is the index indicating the $j$ th triangle facet intersecting the $i$ th layer, $M$ is the total numbers of triangle facets intersect the $i$ th layer, $k$ is the index indicating the $k$ th triangle facet of the STL file, $N$ is the total number of triangle facets of the STL file, $\xi$ is the normal vector of the facet, and $B$ is the building direction.

\subsubsection{Tolerance volume of the peaks and valleys}

The equation for calculating the tolerance volume of peaks can be expressed by Eq. (5) based on the unit of a layer. In fact, it can be further developed into Eqs. (4.1) and (4.2) by combination of two connective triangle facets. The details of the peaks are shown in Fig. 6(b).

Tolerance volume $=0.5$

$$
\cdot\left(\sum_{p=1} a_{1 p} \cdot c_{1 p}+\sum_{p=1} a_{2 p} \cdot c_{2 p}\right)
$$

where

$c_{1 p}=(l-\Delta l) \cdot\left|\cos \theta_{1}\right|$

$c_{2 p}=\Delta l \cdot\left|\cos \left(\pi-\theta_{2}\right)\right|=\Delta l \cdot\left|\cos \theta_{2}\right|$

$\theta_{1}=\frac{\overrightarrow{\boldsymbol{B}} \cdot \vec{\xi}_{1}}{|\overrightarrow{\boldsymbol{B}}|\left|\vec{\xi}_{1}\right|}, \quad \theta_{2}=\frac{\overrightarrow{\boldsymbol{B}} \cdot \vec{\xi}_{2}}{|\overrightarrow{\boldsymbol{B}}|\left|\vec{\xi}_{2}\right|}$

where $1 p$ and $2 p$ are indices indicating the $p$ th connective triangle facet of peak or valley, $l$ is the layer thickness, $\Delta l$ is the thinner layer height where peaks or valleys touch, $c_{1 p}$ and $c_{2 p}$ are the cusp height of the $p$ th peak or valley, and $a_{1 p}, a_{2 p}$ are the inclined areas of the $p$ th peak or valley.

\subsubsection{Tolerance volume of the flat areas}

The equation below is used to calculate the tolerance volume of flat areas as shown in Fig. 6(c):

Tolerance volume $=\sum_{i=1}^{N} \Delta l \cdot A_{i}$

where $i$ is the index indicating the $i$ th flat triangle facet of the STL file, and $N$ is the total number of flat triangle facets of the STL file, $A_{i}$ is the area of the $i$ th flat triangle face, and $\Delta l$ is the thinner layer height.

\subsection{Effective cusp height for post-processing process}

The effective cusp height $\left(\delta^{*}\right)$ for post-processing process can be obtained by the following equation:

effective cusp height $\left(\delta^{*}\right)=\frac{\text { tolerance volume }}{\sum_{i=1}^{N} A_{i}}$

where $i$ is the index indicating the $i$ th flat triangle facet of the STL file, $N$ is the total number of flat triangle facets of the STL file, and $A_{i}$ is the area of the $i$ th flat triangle face. 


\section{Slicing procedures}

The above-slicing strategies are integrated and the slicing procedures are proposed and given in the flowchart shown in Fig. 7.

First of all, input an STL file. Then choose the suitable working tolerance depending on the workpiece function. If the workpiece is determined for industrial mock-up, positive tolerance is specified. If it is to be used as a core, negative tolerance is specified. Then, one layer is added at a time based on the lamination thickness. Starting from the first layer, take any one triangle facet that passes the $z$-plane as the first triangle facet for calculating the intersection point. Next, determine the dot value $(\boldsymbol{D})$ between the normal direction of the triangle facet and working direction. Given the case of positive tolerance, use bottom-up slicing if $\boldsymbol{D} \geq 0$, and top-down slicing if $\boldsymbol{D}<0$. On the contrary, in the case of negative tolerance, use top-down slicing if $\boldsymbol{D} \geq 0$, and bottom-up slicing if $\boldsymbol{D}<0$.

If there are special features in the lamination thickness, the slicing calculations of peaks, valleys, flat areas and fine features, called special feature slicing in Fig. 7, will be used. Bottom-up or top-down slicing is employed. Then, the intersection point obtained is then recorded as one point of the loop. Next, search for the connective triangle facet that passes through the $z$-plane through the common edge between triangle facets. Determine whether the triangle facet is the first triangle facet already sliced. If not, use the aforementioned procedures to locate another intersection point. If yes, connect these intersection points to form a closed loop. Next, search for any triangle facet that passes through the $z$-plane but has not been sliced yet. If any, it means that there is another loop. In this case, select one of the triangle facets as the first triangle facet and find other intersection points using the same method to form a close loop. If none, it indicates that all triangle facets that pass through the $z$-plane have been sliced. Finally, these loops form a $2 \mathrm{D}$ crosssection, which is the slicing intersection curve we are looking for to serve as the working path. Then, add another layer of lamination thickness and calculate the slicing intersection curve of the next layer. Repeat these procedures till the maximum $z$ axis value of the workpiece is completed.

Once the full slicing process is completed, the effective cusp height and tolerance are estimated. Then, the whole slicing procedure is finished.

\section{Experimental verification}

To prove that the above-slicing procedures are correct, a 3D part-an inclined cuboid with peak feature-was selected. The STL file and slicing planes are shown in Fig. 8(a). The front view and side view are shown in Fig. 8(b) and Fig. 8(c), respectively.

Fig. 8(d) shows the 2D cross-section obtained by the intersection of the cuboid and the slicing plane. Two dotted areas denote the cross-section of the part after slicing. The area enclosed by $\mathrm{JKLMNA}_{4} \mathrm{OPEFGHIJ}$ is obtained by the top-down slicing method, while the area enclosed by STUVA ${ }_{5}$ WXQRS is obtained by the bottom-up slicing method. The points $A_{1}, A_{2}, A_{3}$ and $\mathrm{Y}$ are obtained by using the slicing procedure of peak discussed in Section 3.1, and depicted by the block of special features slicing in Fig. 7.

The shaded area enclosed by ITUVA 5 WOPEFGHI is obtained by the negative tolerance slicing method, and the area enclosed by JKLMNA 4 OWXQRSJ is the cross-section obtained by the positive tolerance slicing method. The points $\mathrm{A}_{1}, \mathrm{~A}_{2}, \mathrm{~A}_{3}$ and $\mathrm{Y}$ can be obtained after carrying out "special feature slicing" procedures as given in Fig. 7, and a new area enclosed by $\mathrm{A}_{1} \mathrm{~A}_{2} \mathrm{LMNA}_{4} \mathrm{OA}_{3} \mathrm{YQRSA}_{1}$ region will be formed. The procedures of positive tolerance slicing and fine feature slicing are described as follows.

First of all, choose a triangle facet $\mathrm{A}_{1} \mathrm{~A}_{2} \mathrm{~A}_{6}$ that passes through the slicing plane. The positive value of $\boldsymbol{D}$ is obtained after evaluating the inner product of the normal vector of the triangle facet and the working direction. The exact intersection point $\mathbf{J}$ can be obtained by intersection of the slicing plane and triangle facet $\mathrm{A}_{1} \mathrm{~A}_{2} \mathrm{~A}_{6}$. Due to the specified positive tolerance slicing, the intersection point NONE instead of $\mathbf{J}$ is obtained by the bottom-up slicing method based on Eq. (2). In case of a peak feature, the slicing thickness is set to a smaller value call "fine slice"; when slicing occurs from the smaller $Z$ value upward, peaks occur at the layer where the $\boldsymbol{D}$ value changes from a negative value to a positive one. Then, the intersection point $A_{1}$ instead of NONE is obtained by this method as discussed in Section 3.1.

Then, choose the connective triangle facet $\mathrm{A}_{2} \mathrm{~A}_{6} \mathrm{~A}_{7}$ that passes through the slicing plane through the common edge between triangle facets, and calculate the exact intersection point $\mathrm{K}$ of slicing triangle facet and slicing plane. Due to the specified positive tolerance slicing, the intersection point NONE instead of $\mathrm{K}$ is obtained by bottom-up slicing. Similarly, in case of peaks intersection point $\mathrm{A}_{2}$ instead of NONE is obtained based on the "fine slice" procedure.

Similarly, choose the triangle facets $\mathrm{A}_{7} \mathrm{~A}_{2} \mathrm{~A}_{8}$ and $\mathrm{ABA}_{9}$; then the intersection points $\mathrm{L}$ and $\mathrm{M}$ are obtained by the top-down slicing method based on the negative value of $\boldsymbol{D}$. Similarly, choose the triangle facet $\mathrm{AA}_{9} \mathrm{~B}_{3}$; then the intersection point $\mathrm{N}$ is obtained by the top-down slicing method based on the negative value of $\boldsymbol{D}$. Then, choose the triangle facet $\mathrm{AB}_{1} \mathrm{~A}_{3}$, and calculate the intersection point $\mathrm{A}_{4}$ by the top-down slicing method based on the negative value of $\boldsymbol{D}$. 


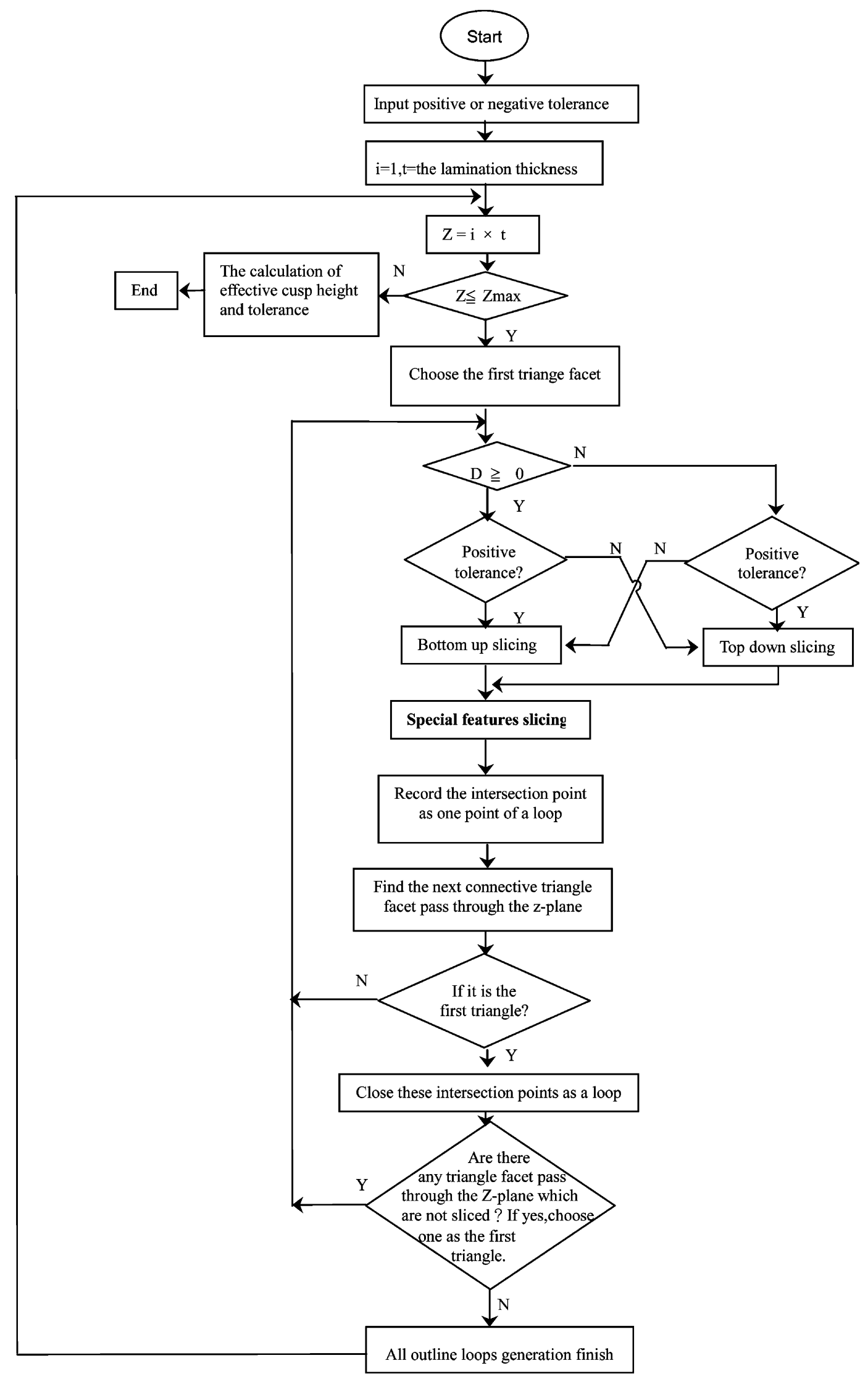

Fig. 7. Slicing procedures. 
(a)

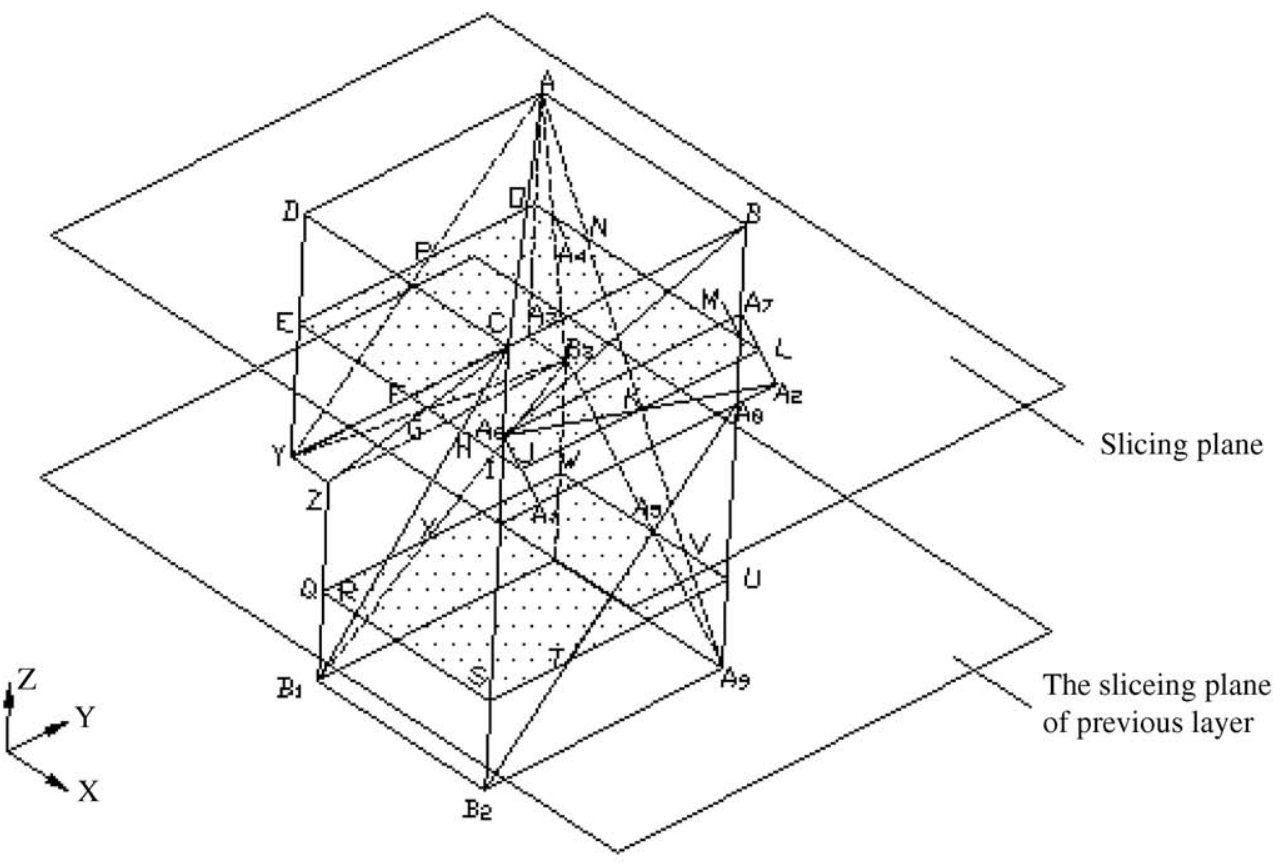

(b)

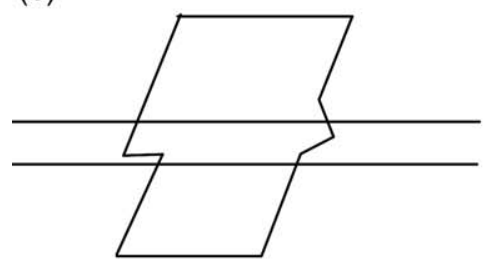

(c)

slice $(\mathrm{i}+1)$

slice ( i )

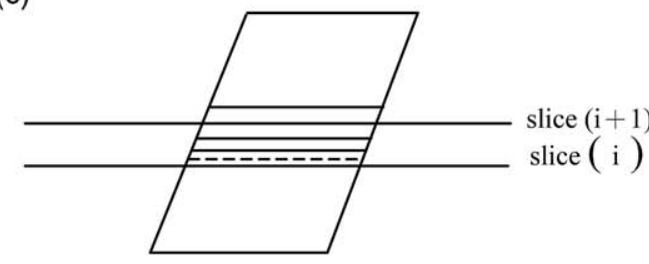

(d)

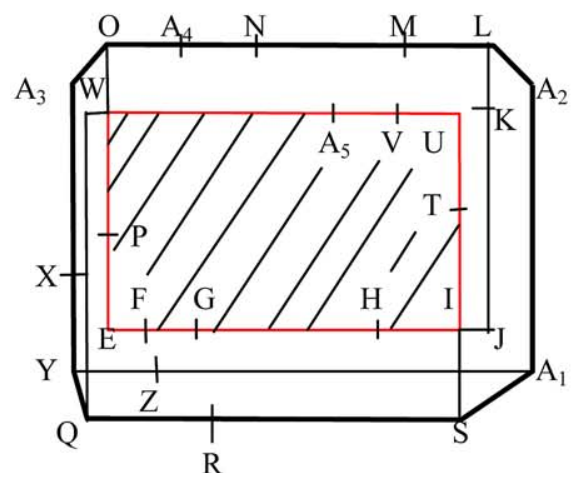

Fig. 8. (a) Schematic diagram of inclined cuboid with peak feature and slicing plane. (b) Front view. (c) Side view. (d) The shaded area denotes the cross-section of the part after negative tolerance slicing. The solid line inside is the cross-section of the part after positive tolerance slicing.

Repeating similar procedures, choose the triangle facets $\mathrm{AA}_{3} \mathrm{Y}$ and $\mathrm{ADY}$; then the intersection points $\mathrm{O}$ and $\mathrm{P}$ are obtained, respectively, by intersection of the slicing plane and the triangle facets. Due to the specified positive tolerance slicing, the intersection points NONE rather than $\mathrm{O}$ and $\mathrm{P}$ will be obtained by the bottom-up slicing method based on $\boldsymbol{D}>0$. In case of this peak feature, the peaks will be obtained by "fine slice", which occurs at the layer where the $\boldsymbol{D}$ value changes from a negative value to a positive one while going from the smaller $Z$ value upward. Therefore, the intersection points $\mathrm{A}_{3}$ and $\mathrm{Y}$ instead of NONE will be obtained.

Then, choose the connective triangle facet CDY and CYZ that passes through the slicing plane through the common edge between triangle facets, and calculate the 
intersection points $\mathrm{E}$ and $\mathrm{F}$ of the triangle facet and slicing lane. Due to the specified positive tolerance slicing, the intersection point NONE instead of $\mathrm{E}$ and $\mathrm{F}$ is obtained by the bottom-up slicing method based on $\boldsymbol{D}>0$. Then, choose the connective triangle facets $\mathrm{CZB}_{1}$ and $\mathrm{CB}_{1} \mathrm{~B}_{2}$ and $\mathrm{A}_{5} \mathrm{~A}_{6} \mathrm{~A}_{1}$; the intersection points, $\mathrm{Q}, \mathrm{R}$ and $\mathrm{S}$ will be obtained by the bottom-up slicing method based on the positive value of $\boldsymbol{D}$. Finally, the region enclosed by points of JKLMNA ${ }_{4} \mathrm{OWXQRSJ}$ as shown in Fig. 8(d) is the cross-section of positive tolerance slicing.

The negative tolerance slicing plane depicted by the shaded region enclosed by points of ITUVA ${ }_{5}$ WOPEFGHI as shown in Fig. 8(c) can be obtained similarly.

\section{Conclusions}

A new slicing method based on uniform thickness, and adopting the inner product of the normal vector of the STL file and the working direction as the judgment rule, is proposed in this paper. Depending on the workpiece function requirement of either positive or negative tolerance, the top-down or bottom-up slicing method is selected to ensure unilateral tolerance over the whole prototype. Furthermore, to reduce error, proper solutions are presented for such geometric shapes as peaks, valleys, flat areas and fine features, which are easily lost, and the amounts of the tolerance volume and effective cusp are estimated for post-processing. Experimental results have shown that there are three major effects of this method. First of all, it improves the disadvantages of under-cut and over-cut existing in the various previously derived RP slicing methods, and the precision of the completed workpiece can be ensured after post-processing procedures. Secondly, the proposed slicing rule for uniform thickness ensures that the entire workpiece possesses either positive or negative tolerance and geometric shapes such as peaks, valleys, flat areas and fine features are retained. Finally, the tolerance volume and effective cusp height needed for waste removal post-processing are calculated.

\section{References}

[1] W. Cheng, F.Y.H. Fuh, A.Y.C. Nee, et al., Multi-objective optimization of part-building orientation in stereolithography, Rapid Prototyping Journal 1 (4) (1995) 12-23.

[2] G.M. Fadel, C. Krischman, Accuracy issues in CAD to RP translations, Rapid Prototyping Journal 2 (2) (1996) 4-17.

[3] A. Dolenc, I. Makela, Slicing procedures for layered manufacturing techniques, Computer-Aided Design 26 (2) (1994) 119 126.

[4] P. Kulkarni, D. Dutta, An accurate slicing procedure for layered manufacturing, Computer-Aided Design 28 (9) (1996) 683-697.

[5] E. Sabourin, S.A. Houser, F.H. Bohn, Adaptive slicing using stepwise uniform refinement, Rapid Prototyping Journal 2 (4) (1996) 20-26.

[6] E. Sabourin, S.A. Houser, F.H. Bohn, Accurate exterior, fast interior layered manufacturing, Rapid Prototyping Journal 3 (2) (1997) 44-52.

[7] R.L. Hope, P.A. Facobs, R.N. Roth, Rapid prototyping with sloping surfaces, Rapid Prototyping Journal 3 (1) (1997) 12-19.

[8] R.L. Hope, R.N. Roth, P.A. Facobs, Adaptive slicing with sloping layer surfaces, Rapid Prototyping Journal 3 (3) (1997) 89-98.

[9] Y.S. Liao, Y.Y. Chiu, A new slicing procedures for rapid prototyping systems, International Journal of Advanced Manufacturing Technology 18 (2000) 579-585.

[10] M. Feygin, S.S. Pak, Laminated object manufacturing apparatus and method, United States Patent, No. 5,876,550, 1999. 